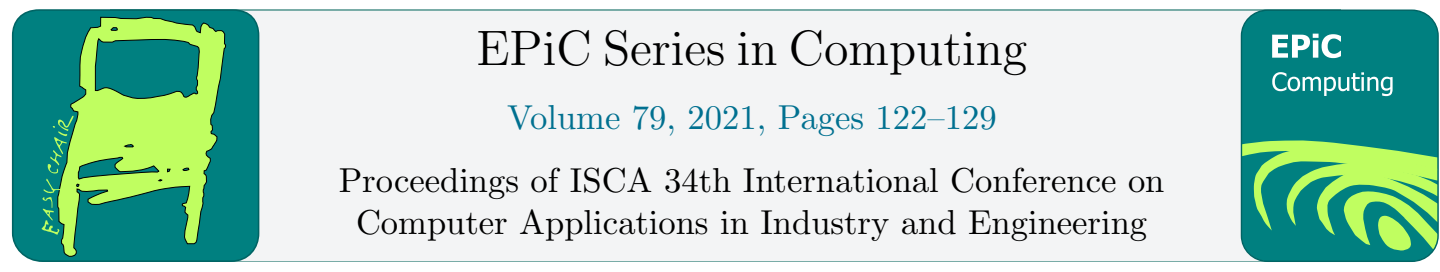

\title{
Efficient Vehicular Communication using Peer to Peer Pyramid Trees
}

\author{
Henry Hexmoor, Shameerpet Tanuja, and Bidyut Gupta * \\ School of Computing \\ Southern Illinois University \\ Carbondale, IL 62901, USA \\ hexmoor, tanuja.shameerpet, bidyut [ [siu.edu]
}

\begin{abstract}
The emerging concept of vehicular communication including with the roadside infrastructure is a promising solution to avoid accidents and providing live traffic data. There is a high demand for the technologies which ensure low latency vehicular communication. Modern vehicles equipped with computing, communication, storage, and sensing capabilities expedite data exchange. To achieve deterministic bounds on data delivery, ability to be established anywhere quickly, as well as efficiency of data query, we describe a novel peer to peer structured overlay model for a cluster of vehicles known as the pyramid tree model.
\end{abstract}

\section{Introduction}

We consider vehicle to vehicle direct communication and with the roadside infrastructure using vehicle onboard units. Peer to peer inter-vehicle communication model provides a facility to avoid accidents and congestions by implementing direct communication among the vehicle nodes. Our current focus is application of pyramid tree (PT) for efficient inter-vehicle communications [4]. A pyramid tree is group of clusters organized in a structured format with the data links between the clusters. The core concepts in formation of a pyramid tree model are clustering the nodes based on their common interest [6]. Pyramid trees can be formed anywhere and with minimal formation time. The predominant benefit of PT is improved bandwidth utilization by reducing duplicate packet generation that is commonly found in vehicular broadcast communication. Vehicular communication requires low latency achieved by PT, independent of the specific vehicles in the cluster. The salient feature of PT is to group the nodes into clusters based on the type of resource constituent nodes have in common such as a shared

${ }^{*}$ Created the first draft of this document 
interest. In this paper we limit ourselves to complete PTs and postpone partial PTs for future consideration. Vehicles are grouped into clusters based on their physical proximity. Section 2 will discuss the architecture of the pyramid tree and broadcast in complete PTs. Section 3 will offer observations and benefits of implementing PT for vehicular communication. Finally, section 4 offers concluding remarks.

\section{Fundamentals}

The VANET has established vehicular communication using on-board wireless communication units (OBU) to facilitate information exchange pertaining to vehicle mobility dynamics and pertinent environmental conditions [3][9][10][11]. OBU equipped vehicles periodically broadcast their mobility dynamics such as coordinates, speed, and direction as well as acceleration and deceleration (i.e., braking). The standard VANET communication adheres to the seven-layer Open-Source Interconnection (OSI) networking standard. VANET communication includes DSRC (Dedicated shortrange communication protocol), C-LTE (mobile wireless communication technology based on cellular networks), and IEEE $802.11 \mathrm{~b}$ (Wi-Fi) [7]. For the non-critical applications, such as traffic information transmission, file download, or internet access, which do not require the high-speed real-time communication, 4G-LTE is the most appropriate. In contrast, for safety critical applications such as collision avoidance and electronic traffic signal control, DSRC is recommended [8]. Our current focus is peer to peer ( $\mathrm{P} 2 \mathrm{P})$ networking overlay protocol at the application layer over the existing network [1][2][5]. Whereas VANET is responsible for establishing communication channels, dynamic route scheduling and maintaining neighboring list for each vehicle and monitoring the bandwidth utilization, P2P is used to locate peers not using IP address but via logical identifiers. The most prominent P2P protocols are BitTorrent, Blockchain, direct connect, Gnutella, web torrent. An unstructured P2P Peer to Peer network is a collection of the nodes that are randomly connected with each other; i.e., P2P overlay links are arbitrarily established not imposing rigid structure on the network (e.g.,. KaZaA, Napster, Gnutella) [1][2]. Unstructured P2P suffers from lack of guaranteed lookups and high volume of traffic and duplicate packets. In contrast, peers in structured P2P are members of specific topologies; lookups are guaranteed with deterministic time bound. Commonly, structured P2P protocols maintain a DHT (Distributed Hash Table) and allows each peer to be responsible for specific part of the content in the network. The popular protocols Chord and CAN are structured P2P protocols. Maintaining DHTs is a tedious task, which does not effectively handle the problem of churn. DHT-based hybrid systems ameliorate shortcomings by combining advantages of structured and unstructured P2P protocols. Our proposed PT is a non-DHT based structured approach that seeks to reduce the complexity involved in maintaining DHTs and churn handling. A node $\mathrm{i}$ in a pyramid tree represents a cluster of nodes interested in a particular resource type Ri. The pyramid is classified to complete and incomplete pyramid trees. A pyramid tree is complete if it has $\mathrm{j}$ number of nodes at each level $\mathrm{j}$. In a pyramid tree, at each level $j$, the number of nodes can be less than $\mathrm{j}$, but cannot be more than $\mathrm{j}$. If the number of nodes at level $\mathrm{j}$ is less than $\mathrm{j}$, the tree is incomplete (i.e., partial PT). We have prototyped the complete pyramid tree model for our vehicular communication model. In a complete pyramid tree, if the number of levels $i \mathrm{j}$, the number of nodes will be $\mathrm{j}^{2}$. In subsequent sections the cluster formation, types of clusters, and broadcast mechanisms are discussed.

Vehicles are grouped into clusters based on their interest or participation in a specific resource. Let us consider the physical neighborhood of a vehicle (i.e., vehicle proximity) be the vehicle's resource, which clusters vehicles in a physical zone grouped together [6]. Each cluster is represented by a 
representative cluster head. The first vehicle V1 entering to a certain physical region R1 is termed as a cluster head. The roadside unit associated with the region R1 delivers the live traffic data to the cluster head. The vehicles $\mathrm{V} 1, \mathrm{~V} 2, \mathrm{v} 3, \mathrm{v} 4 \ldots, \mathrm{vn}$ entering to the cluster $\mathrm{C} 1$ constitute members of the cluster. For illustration, let the range of the cluster be one to fifteen vehicles, where one is the smallest number and fifteen is the largest number of vehicles grouped in the cluster. Let the length of the area spanned by a cluster be limited to 120 feet that is six vehicles with 20 feet per vehicle and 10 feet width (i.e., about 40 meters length and 3 meters width). The total area spanned by a pyramid tree is 1200 square feet (i.e., 120 square meters) and the number of clusters in a pyramid tree is restricted to fifteen. By restricting the number of vehicles and the area of the cluster of the cluster and the area spanned by a cluster, the communication overhead and the latency is significantly curtailed. Next, we outline a formal model and operational algorithms.

Assume a set of vehicles on the road as $\mathrm{V}=\{\mathrm{V} 1, \mathrm{~V} 2, \mathrm{~V} 3 \ldots \mathrm{Vn})$ and the clusters $\mathrm{C}=\{\mathrm{C} 1, \mathrm{C} 2, \mathrm{C} 3$, $\ldots, \mathrm{Cn})$ at different geographical areas $\mathrm{A}=(\mathrm{A} 1, \mathrm{~A} 2, \mathrm{~A} 3, \ldots, \mathrm{An})$. Consider all vehicles are in transit. Every vehicle that reaches the geographical region $\mathrm{Ai}$, is permitted to join cluster $\mathrm{Ci}$.

The first vehicle entering the geographical area $\mathrm{Ai}$ is elected to be the cluster head $(\mathrm{Ch})$ of the cluster $\mathrm{Ci}$. Remaining vehicles in physical proximity of Chi join as members of the cluster $\mathrm{Ci}$. The given cluster head must contain the information of other cluster heads that are present in the tree. Whenever a new cluster head joins, the root cluster head is responsible for broadcasting the information to all the cluster heads in the tree. The algorithm for formation of a cluster is outlined next:

1. Vehicle one initiates the formation of cluster when it reaches Area one.

2. $A \leftarrow$ Set of all the geographic Areas $\left(A_{1}, A_{2}, A_{3} \ldots, A_{n}\right)$

3. $\mathrm{CH} \leftarrow$ Set of the cluster heads in the pyramid tree $\left(\mathrm{C}_{\mathrm{h} 1}, \mathrm{C}_{\mathrm{h} 2}, \mathrm{C}_{\mathrm{h} 3} \ldots . . \mathrm{Chh}_{\mathrm{hn}}\right)$

4. $\mathrm{C} \leftarrow$ Set of clusters in the pyramid tree $\left(\mathrm{C}_{1}, \mathrm{C}_{2}, \mathrm{C}_{3} \ldots \ldots . \mathrm{C}_{\mathrm{n}}\right)$

5. $\mathrm{V} \leftarrow$ Set of vehicles $\left(\mathrm{V} 1, \mathrm{~V}_{2}, \mathrm{~V}_{3} \ldots \ldots \mathrm{V}_{\mathrm{n}}\right)$

6. $\mathrm{N}=$ Number of vehicles in a cluster

7. Initialie $\mathrm{N} ; \mathrm{N} \leftarrow 0$

8. while (true) $/ *$ when a vehicle enters an area

9. while $(\mathrm{N}<16)$

10. if $(\mathrm{N}=0)$ then $/ *$ for cluster $\mathrm{C}_{1}$

11. $\quad \mathrm{V}_{1} \leftarrow \mathrm{CH} 1 . \quad / *$ the first vehicle is the c-head

12. end if

13. $\mathrm{N} \leftarrow \mathrm{N}+1 / *$ when second, third up to 15 vehicles enter the cluster.

14. $\quad \mathrm{V}_{\mathrm{n}} \leftarrow \mathrm{C}_{\mathrm{mi}} / * \mathrm{~V}_{\mathrm{i}}$ is the member of the cluster, $\mathrm{C}_{1}$

15. end while

Vehicles travelling in a road network receive a request (req) from the cluster heads in its proximity to join. Any vehicle can accept the request invitation or reject it within the default time limit. To cope up with the dynamic nature of the vehicular network, the time limit is set to minimal. After the time is elapsed, if the cluster head receives a accept response, a confirmation (conf.) is sent to the vehicle indicating its membership approval in the cluster.

1. cluster head $\left(\mathrm{C}_{\mathrm{hi}}\right)$ sends a request $($ req $)$ to the vehicle $\mathrm{V}$.

2. $\mathrm{V}_{\mathrm{i}} \leftarrow$ Set of vehicles in the cluster $\mathrm{C}_{\mathrm{i}}$

3. Timeout $\leftarrow$ Maximum waiting time to receive a response.

4. The cluster head $\left(\mathrm{C}_{\mathrm{hi}}\right)$ broadcasts a request $(\mathrm{req})$ to all the vehicles in its proximity. region to join.

5. while time_elapsed $<$ Timeout do 
6. Receive response from vehicle $\mathrm{V}$.

7. $\quad V_{\mathrm{m}} \leftarrow \mathrm{V}$

8. Send a confirmation signal conf to vehicle $\mathrm{V}$, indicating its membership in the cluster $\mathrm{C}_{\mathrm{i}}$.

\section{9. end while}

Intra cluster communication as shown in Figure 1 is possible after the cluster is formed the vehicle nodes in the cluster communicate with the cluster head about various themes such as the weather conditions, hospitals, fuel stations, traffic congestions, and accidents, and road blockage. Each vehicle in the cluster is connected to the cluster head of the respective cluster. As each vehicle is connected to every vehicle is connected to the cluster head, the data look-up in intra-cluster is achieved by only one overlay hop. If the vehicle $V_{1}$ member of cluster $C_{1}$ wishes to communicate with vehicle $V_{9}$ member of cluster $\mathrm{C}_{2}$, the only means of communication is through their respective cluster heads $\mathrm{C}_{\mathrm{h} 1}$ and $\mathrm{C}_{\mathrm{h} 2}$. I.e., The vehicles that are members of different clusters are unable to communicate directly. When a vehicle first enters the cluster, it requests the cluster head for information. If the cluster head does not possess the requested information, it broadcast the request to other cluster heads within the pyramid tree.

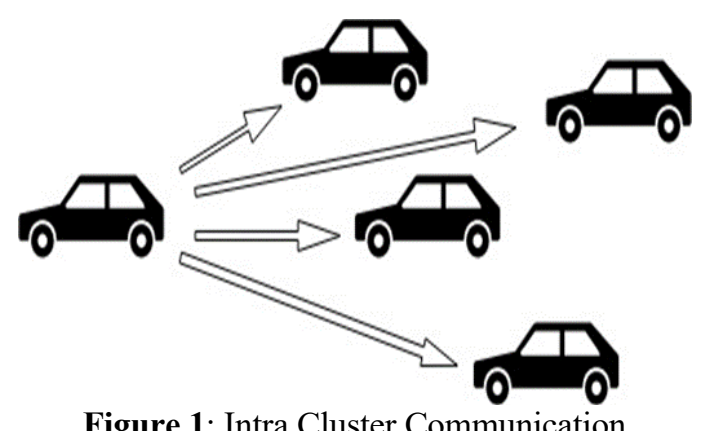

The Inter Cluster communication takes place using the pyramid tree algorithm shown later. Only the cluster heads participate in inter-cluster communication to avoid overhead and efficient bandwidth utilization. Along with providing the requested information to the members of the cluster and broadcasting, maintaining updates to all the cluster heads in the tree, the duties of the cluster head include appointing a member of a cluster as the cluster head and transferring the data, when it departs. The roadside unit then delivers the live data to the new cluster head. The cluster heads are responsible for broadcast updates and emergency information to all clusters in the tree. In Figure 2, only the cluster heads (Labeled 2 and 6) are shown connected.

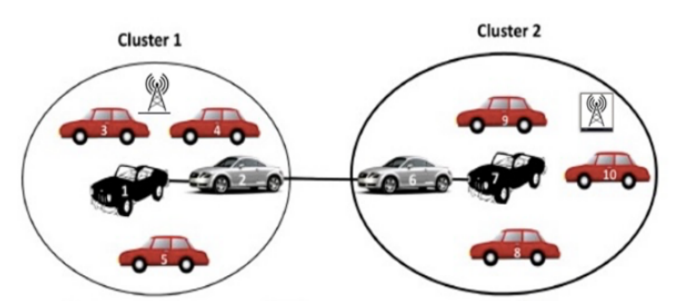

Figure 2: Inter Cluster Communication 


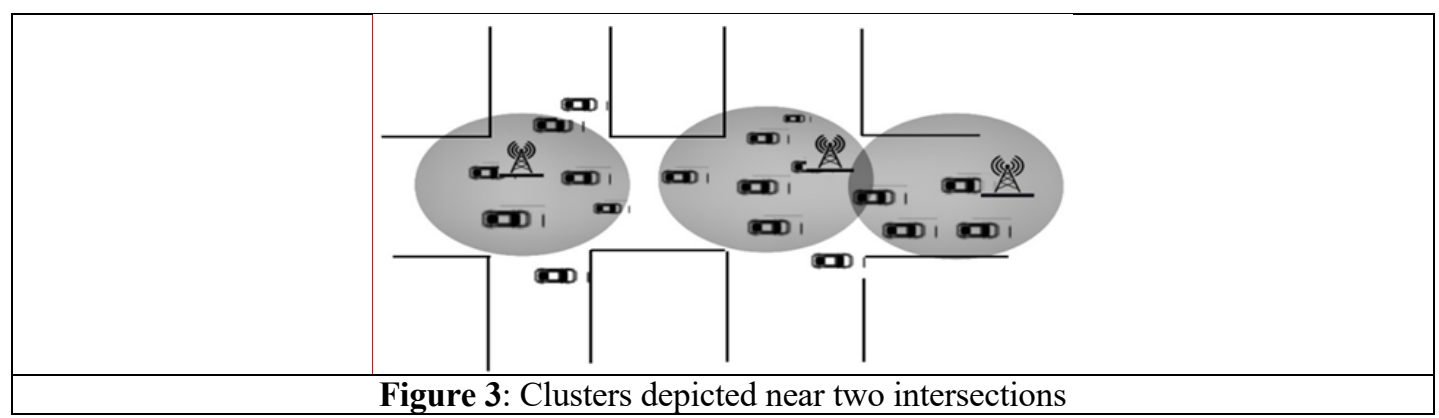

In Figure 3, the road-side units communicate with the cluster heads, cluster head exchanges information with the vehicles, if a vehicle is not in any cluster, i.e., if there are no clusters, in its proximity, the roadside unit communicates with the vehicle. A vehicle can be a member of one or more clusters. I.e., it can retrieve information from one or more cluster heads. The cluster heads of the respective clusters are connected according to the pyramid tree fashion. We can say that a cluster head is a hub of information concerning every vehicle in the cluster, each vehicle updates its actions to the cluster head. The actions include, leaving a highway, entering a parking lot, traffic jams, accidents, etc.

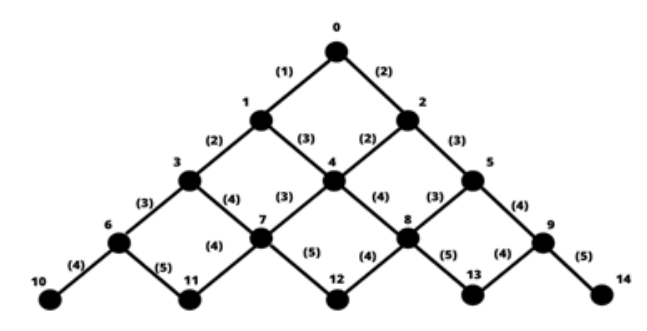

Figure 4: A Complete Pyramid Tree with root labeled 0

Figure 4 illustrates the properties of pyramid trees. As shown in Figure 4, assume $\mathrm{V}$ be the set of vehicles in distinct areas $\mathrm{V}=\left(\mathrm{A}_{1}, \mathrm{~A}_{2}, \mathrm{~A}_{3}, \ldots, \mathrm{A}_{\mathrm{n}}\right)$ and $\mathrm{C}=\left(\mathrm{C}_{1}, \mathrm{C}_{2}, \mathrm{C}_{3}, \ldots, \mathrm{C}_{\mathrm{n}}\right)$ be the set of clusters let $\mathrm{C}_{\mathrm{i}}$ denote the subset of vehicles $V$ in the geographic area $A_{i}$.

The algorithm pyramid Tree formation is outlined next:

1. The Pyramid tree consists of $\mathrm{C}$ clusters.

2. Each Cluster consists of $\mathrm{V}$ vehicles.

3. The first entered vehicle in each cluster is the cluster head $\left(\mathrm{C}_{h}\right)$ and the remaining vehicles of the cluster are cluster members.

4. Each vehicle in the cluster is connected to the cluster head and the Edges among the clusters are formed according to the pyramid tree structure, the edges of the tree denote the logical links among the cluster heads.

5. The communication between $V_{i}$ (belongs to) $C_{i}$ and $V_{j}$ (belongs to) $C_{j}$, takes place only through their respective cluster heads.

6. The new cluster always joins at the leaf level of the pyramid tree. When a new cluster joins, the root cluster head broadcasts update to all the clusters in the pyramid tree.

7. A cluster that does not reside in the left branch or right branch of the root node is the internal cluster. From the above pyramid tree, the clusters $4,7,8,11,12,13$ are internal clusters. 
8. Degree of internal leave node is 2 .

9. Degree of internal non-leaf node is 4 .

Every cluster head in the pyramid maintains the remaining information of the remaining cluster heads of the tree in the form of tables by exchanging the information. When a new cluster head joins the pyramid tree, the root cluster head i.e. Node 0 assigns the next logical address available to the new cluster head, the logical address is assigned in the incremental fashion. The new cluster head will have the greatest logical address and it is linked to the leaf level from left side of the tree. Along with assigning the logical address to the new cluster heads, the root cluster is responsible for broadcasting the "new cluster node entry" information, to the remaining cluster heads in the tree. After receiving the information, each cluster head updates their respective tables. The cluster heads maintain the (IP address, logical address, region) of the remaining cluster heads. The broadcast mechanism involves intra cluster and inter cluster broadcasts.

\section{Implementation and Observations}

To illustrate application of P2P in vehicular communication, we offer a simulation using Netlogo with the interface shown in Figure 5 and intra-cluster communication is illustrated in Figure 6.

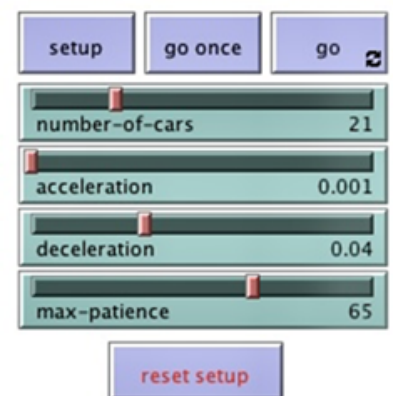

Figure 5: Netlogo Simulation Buttons and Sliders

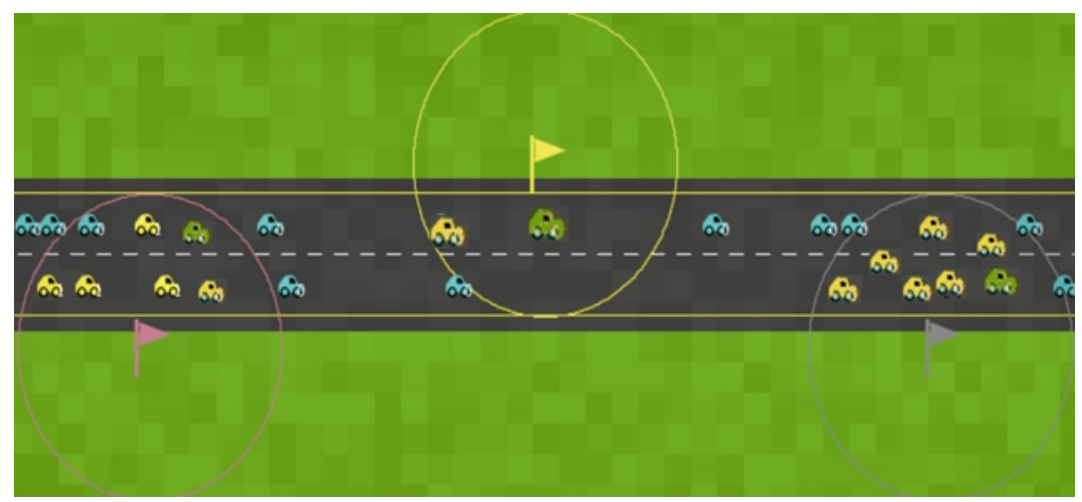

Figure 6: Simulation of Intra-Cluster Communication 


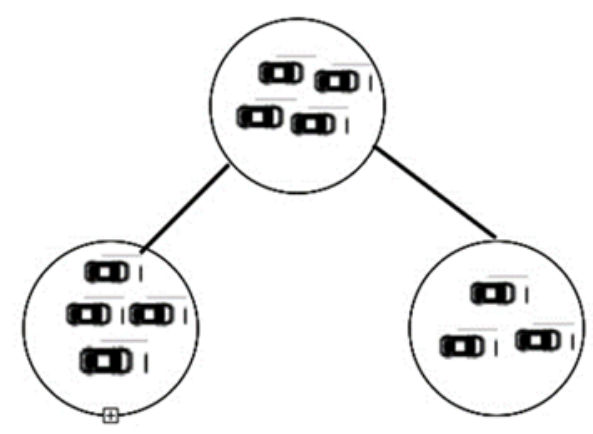

Figure 7: Illustration of broadcast in Pyramid Tree with three clusters

Figure 7 depicts simulation of intra cluster P2P communication, the vehicles sharing information in the cluster [11]. The flags denote data units, which can be base Stations or road-side units. The first vehicle entering the cluster is the cluster head. The cluster head is responsible for sharing information with the vehicles in the cluster. If the number of vehicles in the region is more than six, a separate new cluster is formed. We have simulated intra cluster P2P communication with three clusters. The cluster area and the number of vehicles in the cluster are restricted to decrease the latency and increase the efficiency of the communication. The range of the areas covered by the cluster depend on underlying technologies in use. Let us consider each transmission in a cluster to takes up to $50 \mathrm{~ms}$. Then, the average latency in $4 \mathrm{G} 50 \mathrm{~ms}$ per transmission whereas the latency rate for $5 \mathrm{G}$ ranges in $1 \mathrm{~ms}$ to $26 \mathrm{~ms}$. As we are restricting the number of vehicles to six, the cluster head takes up to $300 \mathrm{~ms}$ to respond to the requests of each per vehicle. In emergencies such as congestions, accidents, brake failures, lane changes, the cluster head broadcasts the information to all the cluster members in up to $50 \mathrm{~ms}$. For each broadcast session there will be $\mathrm{n}-1$ transmissions where $\mathrm{n}$ is the number of vehicles in the cluster. Figure 7 shows inter-cluster P2P communication. Communication is achieved by implementing pyramid tree model. Only the cluster heads participate in intra-cluster communication. We have considered 3 clusters in our simulation. Let us consider that cluster head in the cluster one initiates the broadcast. The broadcast packet is transmitted to the remaining cluster heads, i.e., cluster head two and three in one overlay hop. The cluster head initiating the broadcast is the root of the cluster. Figure 8 illustrates inter-cluster P2P communication. Communication is achieved by implementing pyramid tree model. Only the cluster heads participate in intra-cluster communication. We have considered three clusters in our simulation. Let us consider that the cluster head in the cluster one initiates the broadcast, and the broadcast packet is sent to the remaining cluster heads, i.e., cluster heads two and three in one overlay hop. The cluster head initiating the broadcast is the root of the cluster.

The time complexity of pyramid tree is $\mathbf{O}(\mathbf{n})$ where $\mathrm{n}$ is the number of levels in the pyramid tree. The time complexity of intra-cluster communication with three clusters is $\mathbf{O ( 1 )}$. Let us consider one transmission takes $10 \mathrm{~ms}$. Then then the transmission in a pyramid tree with three clusters takes $10 \mathrm{~ms}$. As the number of clusters increase, the time required increases proportionally, which in turn increases the transmission time in the pyramid tree. For a PT with $\mathrm{N}$ levels, $\mathrm{T}$ time required for a single transmission, the total broadcast time is: $\mathbf{T}_{\mathbf{b}}=\mathbf{N} \times \mathbf{T}$. 


\section{Conclusions}

Pyramid trees are applied to vehicular communication with the properties of deterministic bound on data delivery, efficiency of data query, and location independent, rapid deployment in the real world. We have prototyped a structured peer to peer overlay pyramid tree among a cluster of vehicles. Future work will extend our approach from complete pyramid trees herein to partial pyramid trees.

\section{References}

[1] P. Ganesan, Q. Sun, and H. Garcia-Molina, 2003. Yappers: A peer-to-peer lookup service over arbitrary topology, In Proc. of the IEEE Infocom, IEEE press.

[2] Y. Chawathe, S. Ratnasamy, L. Breslau, N. Lanham, and S. Shenker, 2003. Making Gnutella-like P2P Systems Scalable, In Proc. of the ACM SIGCOMM.

[3] L. Chisalita and N. Shahmehri, 2002, A peer-to-peer approach to vehicular communication for the support of traffic safety applications, In IEEE $5^{\text {th }}$ International Conference on Intelligent Transport Systems, 2002, pp.336-341.

[4] I. Roy, N. Rahimi, S. Kaluvakuri, K. Maddali, B. Gupta and N. Debnath, 2020. Design of Efficient Broadcast Protocol for Pyramid Tree-based P2P Network Architecture, In Proc. 33rd International Conference on Computer Applications in Industry and Engineering (CAINE).

[5] B. Shrestha, D. Niyato, Z. Han and E. Hossain, 2008. Wireless Access in Vehicular Environments Using BitTorrent and Bargaining, In IEEE GLOBECOM 2008 - 2008 IEEE Global Telecommunications Conference, IEEE press.

[6] Y. Kanemaru, S. Matsuura, M. Kakiuchi, S. Noguchi, A. Inomata, and K. Fujikawa, 2013. Vehicle Clustering Algorithm for Sharing Information on Traffic Congestion, In 13th International Conference on ITS Telecommunications (ITST), pp. 38-43, IEEE press.

[7] C. Campolo and A. Molinaro, 2011. On vehicle-to-roadside communications in 802.11p/WAVE VANETs, In IEEE Wireless Communications and Networking Conference, Cancun, Mexico, 2011, pp. 1010-1015.

[8] Wai Chen and Shengwei Cai, Ad hoc peer-to-peer network architecture for Vehicle Safety Communications, 2005. In IEEE Communications Magazine, vol. 43, no. 4, pp. 100-107.

[9] R. Bishop, 2000. Intelligent Vehicle Application world-wide, In IEEE Intelligent Systems, vol. 15, pp. 78-83.

[10] A. Verma, R. Pal, A. Prakash and R. Tripathi, 2018. Information retrieval in two-tier VANET/P2P using RSU as a superpeers, Wireless Communication Technology, Vol 2, Issue 1, WHOICE Pub.

[11] Ono, S., Kikuchi, F., Yamazaki, T., Miyoshi, T., \& Sezaki, K., 2020. An Information Sharing Method Using P2P and V2V communications in VANET. In 2020 IEEE International Conference on Consumer Electronics, IEEE press. 